\title{
Sharp Becker-Stark's type inequalities with power exponential functions
}

Yusuke Nishizawa*

"Correspondence:
yusuke@ube-k.ac.jp
General Education, Ube National
College of Technology, Tokiwadai
2-14-1, Ube, Yamaguchi 755-8555,
Japan

\section{Abstract}

In this paper, we give some inequalities with power exponential functions derived from the left hand side of Becker-Stark's inequality:

$$
\frac{8}{\pi^{2}-4 x^{2}}<\frac{\tan x}{x}<\frac{\pi^{2}}{\pi^{2}-4 x^{2}}
$$

for $0<x<\pi / 2$

MSC: Primary 26D05

Keywords: Becker-Stark's inequality; Bernoulli's inequality; monotonically decreasing function; monotonically increasing function

\section{Introduction}

Becker-Stark's inequality is well known:

$$
\frac{8}{\pi^{2}-4 x^{2}}<\frac{\tan x}{x}<\frac{\pi^{2}}{\pi^{2}-4 x^{2}}
$$

for $0<x<\pi / 2$. The research of Becker-Stark's inequality is one of the active areas in mathematical analysis [1-8]. Recently, Zhu [6] gave the following refinement of Becker-Stark's inequality: For $0<x<\pi / 2$, the inequalities

$$
\frac{8}{\pi^{2}-4 x^{2}}+\frac{2}{\pi^{2}}-\frac{\pi^{2}-9}{6 \pi^{4}}\left(\pi^{2}-4 x^{2}\right)<\frac{\tan x}{x}
$$

and

$$
\frac{\tan x}{x}<\frac{8}{\pi^{2}-4 x^{2}}+\frac{2}{\pi^{2}}-\frac{10-\pi^{2}}{\pi^{4}}\left(\pi^{2}-4 x^{2}\right)
$$

hold, where the constants $-\left(\pi^{2}-9\right) /\left(6 \pi^{4}\right)$ and $-\left(10-\pi^{2}\right) / \pi^{4}$ are the best possible. Moreover, from the right hand side of the inequality (1.1), Chen and Cheung [2] gave the following inequality: For $0<x<\pi / 2$, the inequality

$$
\left(\frac{\pi^{2}}{\pi^{2}-4 x^{2}}\right)^{\theta}<\frac{\tan x}{x}<\left(\frac{\pi^{2}}{\pi^{2}-4 x^{2}}\right)^{\vartheta}
$$

(c) 2015 Nishizawa. This article is distributed under the terms of the Creative Commons Attribution 4.0 International License (http://creativecommons.org/licenses/by/4.0/), which permits unrestricted use, distribution, and reproduction in any medium, provided you give appropriate credit to the original author(s) and the source, provide a link to the Creative Commons license, and indicate if changes were made. 
holds, where the constants $\theta=\pi^{2} / 12$ and $\vartheta=1$ are the best possible. In [5], Sun and Zhu gave a simple proof of the results. The above inequality (1.4) is created based on the right hand side of Becker-Stark's inequality (1.1). However, in this paper we establish some inequalities created based on the left hand side of the inequality (1.1).

\section{Results and discussion}

Motivated by (1.4), in this paper, we give some inequalities with power exponential functions derived from the left hand side of Becker-Stark's inequality (1.1). Since we note that $8 /\left(\pi^{2}-4 x^{2}\right)<1$ for $0<x<\left(\sqrt{\pi^{2}-8}\right) / 2$ and $8 /\left(\pi^{2}-4 x^{2}\right)>1$ for $\left(\sqrt{\pi^{2}-8}\right) / 2<x<\pi / 2$, we obtain the two inequalities as follows.

Theorem 2.1 For $0<x<\left(\sqrt{\pi^{2}-8}\right) / 2$, we have

$$
\left(\frac{8}{\pi^{2}-4 x^{2}}\right)^{\theta}<\frac{\tan x}{x}<\left(\frac{8}{\pi^{2}-4 x^{2}}\right)^{\vartheta(x)}
$$

with the best possible constant $\theta=0$ and the function

$$
\vartheta(x)=\frac{2}{2 x-\sqrt{\pi^{2}-8}}+\frac{2}{\sqrt{\pi^{2}-8}} .
$$

Theorem 2.2 For $\left(\sqrt{\pi^{2}-8}\right) / 2<x<\pi / 2$, we have

$$
\left(\frac{8}{\pi^{2}-4 x^{2}}\right)^{\theta}<\frac{\tan x}{x}<\left(\frac{8}{\pi^{2}-4 x^{2}}\right)^{\vartheta(x)}
$$

with the best possible constant $\theta=1$ and the function

$$
\vartheta(x)=\frac{2}{2 x-\sqrt{\pi^{2}-8}}-\frac{\sqrt{\pi^{2}-8}-\pi+2}{\pi-\sqrt{\pi^{2}-8}} .
$$

From Theorems 2.1 and 2.2, we have the best possible constant $\theta$ such that

$$
\left(\frac{8}{\pi^{2}-4 x^{2}}\right)^{\theta}<\frac{\tan x}{x}
$$

If $0<x<\left(\sqrt{\pi^{2}-8}\right) / 2$, the constant $\theta$ must be $\theta<0$ in order to satisfy $1 \leq \tan x / x<\left(8 /\left(\pi^{2}-\right.\right.$ $\left.\left.4 x^{2}\right)\right)^{\theta}$. On the other hands, if $\left(\sqrt{\pi^{2}-8}\right) / 2<x<\pi / 2$, the constant $\theta$ must be $1<\theta$ in order to satisfy $8 /\left(\pi^{2}-4 x^{2}\right) \leq \tan x / x<\left(8 /\left(\pi^{2}-4 x^{2}\right)\right)^{\theta}$. Here, we obtain the two inequalities as follows.

Theorem 2.3 For $1 / 2<x<\left(\sqrt{\pi^{2}-8}\right) / 2$, we have

$$
\left(\frac{8}{\pi^{2}-4 x^{2}}\right)^{\frac{\vartheta(x)}{8}}<\frac{\tan x}{x},
$$

where the function $\vartheta(x)$ is in Theorem 2.1. 
Corollary 2.4 For $0<x<\pi / 2$, we do not have the best possible constant $\vartheta$ such that

$$
\frac{\tan x}{x}<\left(\frac{8}{\pi^{2}-4 x^{2}}\right)^{\vartheta}
$$

\section{Proofs of main theorems}

\subsection{Proof of Theorem 2.1}

Proof of Theorem 2.1 We set

$$
f(x)=\left(\frac{8}{\pi^{2}-4 x^{2}}\right)^{\frac{2}{2 x-\sqrt{\pi^{2}-8}}+\frac{2}{\sqrt{\pi^{2}-8}}}-\frac{\tan x}{x} .
$$

From

$$
\frac{2}{2 x-\sqrt{\pi^{2}-8}}+\frac{2}{\sqrt{\pi^{2}-8}}<0
$$

for $0<x<\left(\sqrt{\pi^{2}-8}\right) / 2$, by Bernoulli's inequality, we have

$$
\left(\frac{8}{\pi^{2}-4 x^{2}}\right)^{\frac{2}{2 x-\sqrt{\pi^{2}-8}}+\frac{2}{\sqrt{\pi^{2}-8}}}>1+\left(\frac{8}{\pi^{2}-4 x^{2}}-1\right)\left(\frac{2}{2 x-\sqrt{\pi^{2}-8}}+\frac{2}{\sqrt{\pi^{2}-8}}\right) .
$$

By the right hand side of the inequality (1.1), for $0<x<\left(\sqrt{\pi^{2}-8}\right) / 2$,

$$
\begin{aligned}
f(x) & >1+\left(\frac{8}{\pi^{2}-4 x^{2}}-1\right)\left(\frac{2}{2 x-\sqrt{\pi^{2}-8}}+\frac{2}{\sqrt{\pi^{2}-8}}\right)-\frac{\pi^{2}}{\pi^{2}-4 x^{2}} \\
& =\frac{4 x\left(2 \sqrt{\pi^{2}-8} x^{2}-4 x^{2}-\pi^{2} x+8 x+\pi^{2}-8\right)}{\sqrt{\pi^{2}-8}(\pi-2 x)\left(\sqrt{\pi^{2}-8}-2 x\right)(2 x+\pi)} \\
& =\frac{4 x g(x)}{\sqrt{\pi^{2}-8}(\pi-2 x)\left(\sqrt{\pi^{2}-8}-2 x\right)(2 x+\pi)}
\end{aligned}
$$

where

$$
g(x)=2 \sqrt{\pi^{2}-8} x^{2}-4 x^{2}-\pi^{2} x+8 x+\pi^{2}-8 .
$$

From $\sqrt{\pi^{2}-8}-2 x>0$ for $0<x<\left(\sqrt{\pi^{2}-8}\right) / 2$, it suffices to show that

$$
g(x)>0
$$

Here, the derivative of $g(x)$ is

$$
g^{\prime}(x)=8-\pi^{2}+4\left(\sqrt{\pi^{2}-8}-2\right) x
$$

By $8-\pi^{2}<0$ and $\sqrt{\pi^{2}-8}-2<0$, we have $g^{\prime}(x)<0$ for any $0<x<\left(\sqrt{\pi^{2}-8}\right) / 2$. Since $g(x)$ is strictly decreasing for $0<x<\left(\sqrt{\pi^{2}-8}\right) / 2$, we have

$$
g(x)>g\left(\frac{\sqrt{\pi^{2}-8}}{2}\right)=0 .
$$


Therefore, we can get

$$
\frac{\tan x}{x}<\left(\frac{8}{\pi^{2}-4 x^{2}}\right)^{\vartheta(x)}
$$

where

$$
\vartheta(x)=\frac{2}{2 x-\sqrt{\pi^{2}-8}}+\frac{2}{\sqrt{\pi^{2}-8}} .
$$

Since $\tan x / x$ is strictly increasing for $0<x<\pi / 2$, we have

$$
\frac{8}{\pi^{2}-4 x^{2}}<1<\frac{\tan x}{x}
$$

for any $0<x<\left(\sqrt{\pi^{2}-8}\right) / 2$. Hence, for $0<x<\left(\sqrt{\pi^{2}-8}\right) / 2$, we obtain

$$
\left(\frac{8}{\pi^{2}-4 x^{2}}\right)^{\theta}<\frac{\tan x}{x}<\left(\frac{8}{\pi^{2}-4 x^{2}}\right)^{\vartheta(x)},
$$

where the constant $\theta=0$. Since $\vartheta(x)$ is strictly decreasing for $0<x<\left(\sqrt{\pi^{2}-8}\right) / 2$ and

$$
\vartheta(x)<\vartheta(0)=0
$$

the constant $\theta=0$ is the best possible. Therefore, the proof of Theorem 2.1 is complete.

\subsection{Proof of Theorem 2.2}

Proof of Theorem 2.2 We set

$$
f(x)=\left(\frac{8}{\pi^{2}-4 x^{2}}\right)^{\frac{2}{2 x-\sqrt{\pi^{2}-8}}-\frac{\sqrt{\pi^{2}-8}-\pi+2}{\pi-\sqrt{\pi^{2}-8}}}-\frac{\tan x}{x} .
$$

From

$$
\frac{2}{2 x-\sqrt{\pi^{2}-8}}-\frac{\sqrt{\pi^{2}-8}-\pi+2}{\pi-\sqrt{\pi^{2}-8}}>1
$$

for $\left(\sqrt{\pi^{2}-8}\right) / 2<x<\pi / 2$, by Bernoulli's inequality, we have

$$
\begin{aligned}
& \left(\frac{8}{\pi^{2}-4 x^{2}}\right)^{\frac{2}{2 x-\sqrt{\pi^{2}-8}}-\frac{\sqrt{\pi^{2}-8}-\pi+2}{\pi-\sqrt{\pi^{2}-8}}} \\
& \quad>1+\left(\frac{8}{\pi^{2}-4 x^{2}}-1\right)\left(\frac{2}{2 x-\sqrt{\pi^{2}-8}}-\frac{\sqrt{\pi^{2}-8}-\pi+2}{\pi-\sqrt{\pi^{2}-8}}\right) .
\end{aligned}
$$


By the inequality (1.3), for $\left(\sqrt{\pi^{2}-8}\right) / 2<x<\pi / 2$,

$$
\begin{aligned}
f(x)> & +\left(\frac{8}{\pi^{2}-4 x^{2}}-1\right)\left(\frac{2}{2 x-\sqrt{\pi^{2}-8}}-\frac{\sqrt{\pi^{2}-8}-\pi+2}{\pi-\sqrt{\pi^{2}-8}}\right) \\
& -\left(\frac{8}{\pi^{2}-4 x^{2}}+\frac{2}{\pi^{2}}-\frac{10-\pi^{2}}{\pi^{4}}\left(\pi^{2}-4 x^{2}\right)\right) \\
= & \frac{g(x)}{\pi^{4}\left(\sqrt{\pi^{2}-8}-\pi\right)\left(\sqrt{\pi^{2}-8}-2 x\right)(2 x+\pi)},
\end{aligned}
$$

where

$$
\begin{aligned}
g(x)= & 16 \pi^{3} x^{4}-16 \pi^{2} \sqrt{\pi^{2}-8} x^{4}+160 \sqrt{\pi^{2}-8} x^{4}-160 \pi x^{4} \\
& +16 \pi^{4} x^{3}-224 \pi^{2} x^{3}-16 \pi^{3} \sqrt{\pi^{2}-8} x^{3}+160 \pi \sqrt{\pi^{2}-8} x^{3}+640 x^{3} \\
& +8 \pi^{4} x^{2}-40 \pi^{3} x^{2}+8 \pi^{2} \sqrt{\pi^{2}-8} x^{2}+320 \pi x^{2} \\
& -4 \pi^{6} x+48 \pi^{4} x-128 \pi^{2} x+4 \pi^{5} \sqrt{\pi^{2}-8} x \\
& -32 \pi^{3} \sqrt{\pi^{2}-8} x-\pi^{7}-2 \pi^{6}+16 \pi^{5}+16 \pi^{4} \\
& -64 \pi^{3}+\pi^{6} \sqrt{\pi^{2}-8}-8 \pi^{4} \sqrt{\pi^{2}-8} .
\end{aligned}
$$

From $\left(\sqrt{\pi^{2}-8}-\pi\right)\left(\sqrt{\pi^{2}-8}-2 x\right)>0$ for $\left(\sqrt{\pi^{2}-8}\right) / 2<x<\pi / 2$, it suffices to show that

$$
g(x)>0
$$

We have the derivatives

$$
\begin{aligned}
g^{\prime}(x)= & 4\left(16 \pi^{3} x^{3}-16 \pi^{2} \sqrt{\pi^{2}-8} x^{3}+160 \sqrt{\pi^{2}-8} x^{3}-160 \pi x^{3}\right. \\
& +12 \pi^{4} x^{2}-168 \pi^{2} x^{2}-12 \pi^{3} \sqrt{\pi^{2}-8} x^{2}+120 \pi \sqrt{\pi^{2}-8} x^{2}+480 x^{2} \\
& +4 \pi^{4} x-20 \pi^{3} x+4 \pi^{2} \sqrt{\pi^{2}-8} x+160 \pi x \\
& \left.-\pi^{6}+12 \pi^{4}-32 \pi^{2}+\pi^{5} \sqrt{\pi^{2}-8}-8 \pi^{3} \sqrt{\pi^{2}-8}\right) \\
= & 4 h(x)
\end{aligned}
$$

and

$$
\begin{aligned}
h^{\prime}(x)= & 4\left(12 \pi^{3} x^{2}-12 \pi^{2} \sqrt{\pi^{2}-8} x^{2}+120 \sqrt{\pi^{2}-8} x^{2}-120 \pi x^{2}\right. \\
& +6 \pi^{4} x-84 \pi^{2} x-6 \pi^{3} \sqrt{\pi^{2}-8} x+60 \pi \sqrt{\pi^{2}-8} x+240 x \\
& \left.+\pi^{4}-5 \pi^{3}+\pi^{2} \sqrt{\pi^{2}-8}+40 \pi\right) \\
= & 4 k(x) .
\end{aligned}
$$

From

$$
-12\left(\pi^{2}-10\right)\left(\sqrt{\pi^{2}-8}-\pi\right) \cong-2.77627<0
$$


and

$$
-6\left(\pi^{2}-10\right)\left(\pi \sqrt{\pi^{2}-8}-\pi^{2}+4\right) \cong-1.23145<0,
$$

we have

$$
\begin{aligned}
k(x)= & -12\left(\pi^{2}-10\right)\left(\sqrt{\pi^{2}-8}-\pi\right) x^{2}-6\left(\pi^{2}-10\right)\left(\pi \sqrt{\pi^{2}-8}-\pi^{2}+4\right) x \\
& +\pi^{2} \sqrt{\pi^{2}-8}+\pi^{4}-5 \pi^{3}+40 \pi \\
> & -12\left(\pi^{2}-10\right)\left(\sqrt{\pi^{2}-8}-\pi\right)\left(\frac{\pi}{2}\right)^{2}-6\left(\pi^{2}-10\right)\left(\pi \sqrt{\pi^{2}-8}-\pi^{2}+4\right)\left(\frac{\pi}{2}\right) \\
& +\pi^{2} \sqrt{\pi^{2}-8}+\pi^{4}-5 \pi^{3}+40 \pi \\
\cong & 72.7519 .
\end{aligned}
$$

Since $h(x)$ is strictly increasing for $\left(\sqrt{\pi^{2}-8}\right) / 2<x<\pi / 2$, we have

$$
h(x)>h\left(\frac{\sqrt{\pi^{2}-8}}{2}\right) \cong 191.598 .
$$

Thus, $g(x)$ is strictly increasing for $\left(\sqrt{\pi^{2}-8}\right) / 2<x<\pi / 2$ and we have

$$
g(x)>g\left(\frac{\sqrt{\pi^{2}-8}}{2}\right)=0 .
$$

Therefore, we can get

$$
\frac{\tan x}{x}<\left(\frac{8}{\pi^{2}-4 x^{2}}\right)^{\vartheta(x)}
$$

where

$$
\vartheta(x)=\frac{2}{2 x-\sqrt{\pi^{2}-8}}-\frac{\sqrt{\pi^{2}-8}-\pi+2}{\pi-\sqrt{\pi^{2}-8}} .
$$

Since we have

$$
1<\frac{8}{\pi^{2}-4 x^{2}}<\frac{\tan x}{x}
$$

for any $\left(\sqrt{\pi^{2}-8}\right) / 2<x<\pi / 2$, we obtain

$$
\left(\frac{8}{\pi^{2}-4 x^{2}}\right)^{\theta}<\frac{\tan x}{x}<\left(\frac{8}{\pi^{2}-4 x^{2}}\right)^{\vartheta(x)},
$$

where the constant $\theta=1$. Since $\vartheta(x)$ is strictly decreasing for $\left(\sqrt{\pi^{2}-8}\right) / 2<x<\pi / 2$ and

$$
\vartheta(x)>\vartheta\left(\frac{\pi}{2}\right)=1,
$$

the constant $\theta=1$ is the best possible. Hence, the proof of Theorem 2.2 is complete. 


\subsection{Proof of Theorem 2.3 and Corollary 2.4}

We need two lemmas to prove Theorem 2.3.

Lemma 3.1 For $-1 / 5<t<0$, we have

$$
\ln (t+1)>\frac{9}{8} t .
$$

Proof We set

$$
f(x)=\ln (t+1)-\frac{9}{8} t,
$$

then

$$
f^{\prime}(t)=-\frac{9 t+1}{8(t+1)}
$$

From $f^{\prime}(t)>0$ for $-1 / 5<t<-1 / 9$ and $f^{\prime}(t)<0$ for $-1 / 9<t<0, f(t)$ is strictly increasing for $-1 / 5<t<-1 / 9$ and $f(t)$ is strictly decreasing for $-1 / 9<t<0$. Since

$$
f\left(-\frac{1}{5}\right)=\frac{9}{40}-\ln \left(\frac{5}{4}\right) \cong 0.00185645
$$

and

$$
f(0)=0 \text {, }
$$

we can get $f(t)>0$ for $-1 / 5<t<0$.

Lemma 3.2 For $0<s<1 / 5$, we have

$$
\ln (s+1)>\frac{8}{9} s
$$

Proof We set

$$
f(s)=\ln (s+1)-\frac{8}{9} s,
$$

then

$$
f^{\prime}(s)=-\frac{8 s-1}{9(s+1)} .
$$

From $f^{\prime}(s)>0$ for $0<s<1 / 8$ and $f^{\prime}(s)<0$ for $1 / 8<s<1 / 5, f(s)$ is strictly increasing for $0<s<1 / 8$ and $f(s)$ is strictly decreasing for $1 / 8<s<1 / 5$. Since

$$
f\left(\frac{1}{5}\right)=\ln \left(\frac{6}{5}\right)-\frac{8}{45} \cong 0.00454378
$$

and

$$
f(0)=0,
$$

we can get $f(s)>0$ for $0<s<1 / 5$. 
Nishizawa Journal of Inequalities and Applications ( 2015) 2015:402

Page 8 of 11

Proof of Theorem 2.3 We set

$$
\begin{aligned}
f(x) & =\ln \frac{\tan x}{x}-\left(\frac{\vartheta(x)}{8}\right) \ln \frac{8}{\pi^{2}-4 x^{2}} \\
& =\ln \frac{\tan x}{x}-\left(\frac{1}{8 x-4 \sqrt{\pi^{2}-8}}+\frac{1}{4 \sqrt{\pi^{2}-8}}\right) \ln \frac{8}{\pi^{2}-4 x^{2}} .
\end{aligned}
$$

If

$$
t=-1+\frac{8}{\pi^{2}-4 x^{2}}
$$

then $-11 / 100<t<0$ for $1 / 2<x<\left(\sqrt{\pi^{2}-8}\right) / 2$, by Lemma 3.1, we can get

$$
\ln \frac{8}{\pi^{2}-4 x^{2}}>\frac{9}{8}\left(-1+\frac{8}{\pi^{2}-4 x^{2}}\right) .
$$

If

$$
s=-1+\frac{8}{\pi^{2}-4 x^{2}}+\frac{2}{\pi^{2}}-\frac{\pi^{2}-9}{6 \pi^{4}}\left(\pi^{2}-4 x^{2}\right),
$$

then $0<s<1 / 5$ for $1 / 2<x<\left(\sqrt{\pi^{2}-8}\right) / 2$, by Lemma 3.2 and the inequality (1.2), we can get

$$
\begin{aligned}
\ln \frac{\tan x}{x} & >\ln \left(\frac{8}{\pi^{2}-4 x^{2}}+\frac{2}{\pi^{2}}-\frac{\pi^{2}-9}{6 \pi^{4}}\left(\pi^{2}-4 x^{2}\right)\right) \\
& >\frac{8}{9}\left(-1+\frac{8}{\pi^{2}-4 x^{2}}+\frac{2}{\pi^{2}}-\frac{\pi^{2}-9}{6 \pi^{4}}\left(\pi^{2}-4 x^{2}\right)\right) .
\end{aligned}
$$

Since

$$
\frac{1}{8 x-4 \sqrt{\pi^{2}-8}}+\frac{1}{4 \sqrt{\pi^{2}-8}}<0
$$

and

$$
\frac{9}{8}\left(-1+\frac{8}{\pi^{2}-4 x^{2}}\right)<\ln \frac{8}{\pi^{2}-4 x^{2}}<0
$$

for $1 / 2<x<\left(\sqrt{\pi^{2}-8}\right) / 2$, we obtain

$$
\begin{aligned}
f(x)> & \frac{8}{9}\left(-1+\frac{8}{\pi^{2}-4 x^{2}}+\frac{2}{\pi^{2}}-\frac{\pi^{2}-9}{6 \pi^{4}}\left(\pi^{2}-4 x^{2}\right)\right) \\
& -\left(\frac{1}{8 x-4 \sqrt{\pi^{2}-8}}+\frac{1}{4 \sqrt{\pi^{2}-8}}\right) \times \frac{9}{8}\left(-1+\frac{8}{\pi^{2}-4 x^{2}}\right) \\
= & \frac{g(x)}{432 \pi^{4} \sqrt{\pi^{2}-8}(\pi-2 x)\left(\sqrt{\pi^{2}-8}-2 x\right)(\pi+2 x)},
\end{aligned}
$$


where

$$
\begin{aligned}
g(x)= & -18,432 \sqrt{\pi^{2}-8} x^{5}+2,048 \pi^{2} \sqrt{\pi^{2}-8} x^{5} \\
& -73,728 x^{4}+17,408 \pi^{2} x^{4}-1,024 \pi^{4} x^{4} \\
& +972 \pi^{4} x^{3}+15,360 \pi^{2} \sqrt{\pi^{2}-8} x^{3}-4,096 \pi^{4} \sqrt{\pi^{2}-8} x^{3} \\
& +61,440 \pi^{2} x^{2}-24,064 \pi^{4} x^{2}+2,048 \pi^{6} x^{2} \\
& +1,944 \pi^{4} x-243 \pi^{6} x-8,832 \pi^{4} \sqrt{\pi^{2}-8} x+896 \pi^{6} \sqrt{\pi^{2}-8} x \\
& -35,328 \pi^{4}+8,000 \pi^{6}-448 \pi^{8} .
\end{aligned}
$$

It suffices to show that $g(x)>0$ for $1 / 2<x<\left(\sqrt{\pi^{2}-8}\right) / 2$. We have derivatives

$$
\begin{aligned}
g^{\prime}(x)= & -92,160 \sqrt{\pi^{2}-8} x^{4}+10,240 \pi^{2} \sqrt{\pi^{2}-8} x^{4} \\
& -294,912 x^{3}+69,632 \pi^{2} x^{3}-4,096 \pi^{4} x^{3} \\
& +2,916 \pi^{4} x^{2}+46,080 \pi^{2} \sqrt{\pi^{2}-8} x^{2}-12,288 \pi^{4} \sqrt{\pi^{2}-8} x^{2} \\
& +122,880 \pi^{2} x-48,128 \pi^{4} x+4,096 \pi^{6} x \\
& +1,944 \pi^{4}-243 \pi^{6}-8,832 \pi^{4} \sqrt{\pi^{2}-8}+896 \pi^{6} \sqrt{\pi^{2}-8}, \\
g^{\prime \prime}(x)= & 8\left(-46,080 \sqrt{\pi^{2}-8} x^{3}+5,120 \pi^{2} \sqrt{\pi^{2}-8} x^{3}\right. \\
& -110,592 x^{2}+26,112 \pi^{2} x^{2}-1,536 \pi^{4} x^{2} \\
& +729 \pi^{4} x+11,520 \pi^{2} \sqrt{\pi^{2}-8} x-3,072 \pi^{4} \sqrt{\pi^{2}-8} x \\
& \left.+15,360 \pi^{2}-6,016 \pi^{4}+512 \pi^{6}\right) \\
= & 8 h(x),
\end{aligned}
$$

and

$$
\begin{aligned}
\frac{h^{\prime}(x)}{3}= & -46,080 \sqrt{\pi^{2}-8} x^{2}+5,120 \pi^{2} \sqrt{\pi^{2}-8} x^{2} \\
& -73,728 x+17,408 \pi^{2} x-1,024 \pi^{4} x \\
& +243 \pi^{4}+3,840 \pi^{2} \sqrt{\pi^{2}-8}-1,024 \pi^{4} \sqrt{\pi^{2}-8} \\
< & -46,080 \sqrt{\pi^{2}-8}\left(\frac{1}{2}\right)^{2}+5,120 \pi^{2} \sqrt{\pi^{2}-8}\left(\frac{1}{2} \sqrt{\pi^{2}-8}\right)^{2} \\
& -73,728\left(\frac{1}{2}\right)+17,408 \pi^{2}\left(\frac{1}{2} \sqrt{\pi^{2}-8}\right)-1,024 \pi^{4}\left(\frac{1}{2}\right) \\
& +243 \pi^{4}+3,840 \pi^{2} \sqrt{\pi^{2}-8}-1,024 \pi^{4} \sqrt{\pi^{2}-8} \\
= & -147,456+22,528 \pi^{2}-768 \pi^{6}+\pi^{4}\left(5,632+\frac{729}{2} \sqrt{\pi^{2}-8}\right) \\
\cong & -13,629.3 .
\end{aligned}
$$

Thus, $h(x)$ is strictly decreasing for $1 / 2<x<\left(\sqrt{\pi^{2}-8}\right) / 2$. From

$$
h\left(\frac{1}{2}\right) \cong-33,392,
$$


we have $g^{\prime \prime}(x)<0$ for $1 / 2<x<\left(\sqrt{\pi^{2}-8}\right) / 2$. Therefore, $g^{\prime}(x)$ is strictly decreasing for $x_{1}<$ $x<\left(\sqrt{\pi^{2}-8}\right) / 2$. From

$$
g^{\prime}\left(\frac{1}{2}\right) \cong 5,734.6
$$

and

$$
g^{\prime}\left(\frac{\sqrt{\pi^{2}-8}}{2}\right) \cong-67,578
$$

there exists uniquely a real number $x_{1}$ with $1 / 2<x_{1}<\left(\sqrt{\pi^{2}-8}\right) / 2$ such that $g^{\prime}\left(x_{1}\right)=0$. Hence, $g(x)$ is strictly increasing for $1 / 2<x<x_{1}$ and $g(x)$ is strictly decreasing for $x_{1}<x<$ $\left(\sqrt{\pi^{2}-8}\right) / 2$. From

$$
g\left(\frac{1}{2}\right) \cong 4,939
$$

and

$$
g\left(\frac{\sqrt{\pi^{2}-8}}{2}\right)=0
$$

we can get $g(x)>0$ for $1 / 2<x<\left(\sqrt{\pi^{2}-8}\right) / 2$. Hence, the proof of Theorem 2.3 is complete.

Proof of Corollary 2.4 By Theorem 2.3, for $1 / 2<x<\left(\sqrt{\pi^{2}-8}\right) / 2$, we have the following:

$$
\begin{aligned}
\frac{\ln \frac{\tan x}{x}}{\ln \frac{8}{\pi^{2}-4 x^{2}}} & <\frac{1}{8 x-4 \sqrt{\pi^{2}-8}}+\frac{1}{4 \sqrt{\pi^{2}-8}} \\
& =\left(-\frac{1}{4}\right)\left(\frac{x}{\sqrt{\pi^{2}-8}}\right)\left(\frac{1}{\frac{\sqrt{\pi^{2}-8}}{2}-x}\right) .
\end{aligned}
$$

Therefore

$$
\lim _{x \rightarrow\left(\sqrt{\pi^{2}-8}\right) / 2-0} \frac{\ln \frac{\tan x}{x}}{\ln \frac{8}{\pi^{2}-4 x^{2}}}=-\infty .
$$

The proof of Corollary 2.4 is complete.

\section{Conclusions}

In this paper, we gave four inequalities derived from the left hand side of Becker-Stark's inequality (1.1), which are natural generalizations of the inequality (1.1). Since the value of $8 /\left(\pi^{2}-4 x^{2}\right)$ is less than 1 for $0<x<\left(\sqrt{\pi^{2}-8}\right) / 2$ and the value of $8 /\left(\pi^{2}-4 x^{2}\right)$ is larger than 1 for $\left(\sqrt{\pi^{2}-8}\right) / 2<x<\pi / 2$, we established the inequalities in Theorems 2.1 and 2.2 . By Theorem 2.3, we obtained Corollary 2.4 immediately. 


\section{Acknowledgements}

The author would like to thank Professor Mitsuhiro Miyagi and the referees for their helpful suggestions and good advice.

Received: 29 June 2015 Accepted: 6 December 2015 Published online: 18 December 2015

\section{References}

1. Anderson, GD, Vamanamurthy, MK, Vuorinen, M: Inequalities for quasiconformal mappings in space. Pac. J. Math. 160(1), 1-18 (1993)

2. Chen, C-P, Cheung, W-S: Sharp Cusa and Becker-Stark inequalities. J. Inequal. Appl. 2011, Article ID 136 (2011)

3. Debnath, L, Mortici, C, Zhu, L: Refinements of Jordan-Steckin and Becker-Stark inequalities. Results Math. 67(1-2), 207-215 (2015)

4. Mitrinović, DS: Analytic Inequalities. Springer, Berlin (1970)

5. Sun, Z-J, Zhu, L: Simple proofs of the Cusa-Huygens-type and Becker-Stark-type inequalities. J. Math. Inequal. 7(4), 563-567 (2013)

6. Zhu, L: A refinement of the Becker-Stark inequalities. Math. Notes 93(3-4), 421-425 (2013)

7. Zhu, L: Sharp Becker-Stark-type inequalities for Bessel functions. J. Inequal. Appl. 2010, Article ID 838740 (2010)

8. Zhu, L, Hua, J: Sharpening the Becker-Stark inequalities. J. Inequal. Appl. 2010, Article ID 931275 (2010)

\section{Submit your manuscript to a SpringerOpen ${ }^{\circ}$ journal and benefit from:}

- Convenient online submission

Rigorous peer review

- Immediate publication on acceptance

Open access: articles freely available online

- High visibility within the field

- Retaining the copyright to your article 